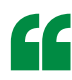

grid cells may

encode spatial

locations

even in a non-

moving animal

\title{
Monkey brains make space for grid cells
}

Grid cells fire when an animal is at specific locations in an environment - a grid cell's so called firing fields. They derive their name from the grid-like pattern in which these firing fields are organized. So far, grid cells have only been identified in the entorhinal cortex (ERC) in rodents and bats; only indirect evidence from a functional MRI study in humans has supported the existence of such cells in primates. Now, Buffalo and colleagues show, in a single-neuron recording study, that grid cells exist in macaque monkeys.

Because it is difficult to record from single neurons in moving monkeys, the authors used three head-fixed monkeys. The monkeys visually explored consecutive complex images presented on a screen (each image was shown twice) while the authors tracked their eye gaze and simultaneously recorded from a large number of neurons in the ERC.

Several neurons in the posterior ERC fired when the monkey's eye gaze was directed at multiple, specific locations on an image. A calculation of the 'gridness' score for each recorded neuron - a measure of the spatial regularity of their firing fields - revealed that over $10 \%$ of recorded neurons in the posterior ERC could be defined as grid cells. The firing fields of each grid cell remained the same throughout the experiment; that is, they were stable and independent of the content of the images on the screen.

The proportion of grid cells in the anterior ERC was smaller than that in the posterior ERC, whereas repetition suppression - the phenomenon that a neuron responds

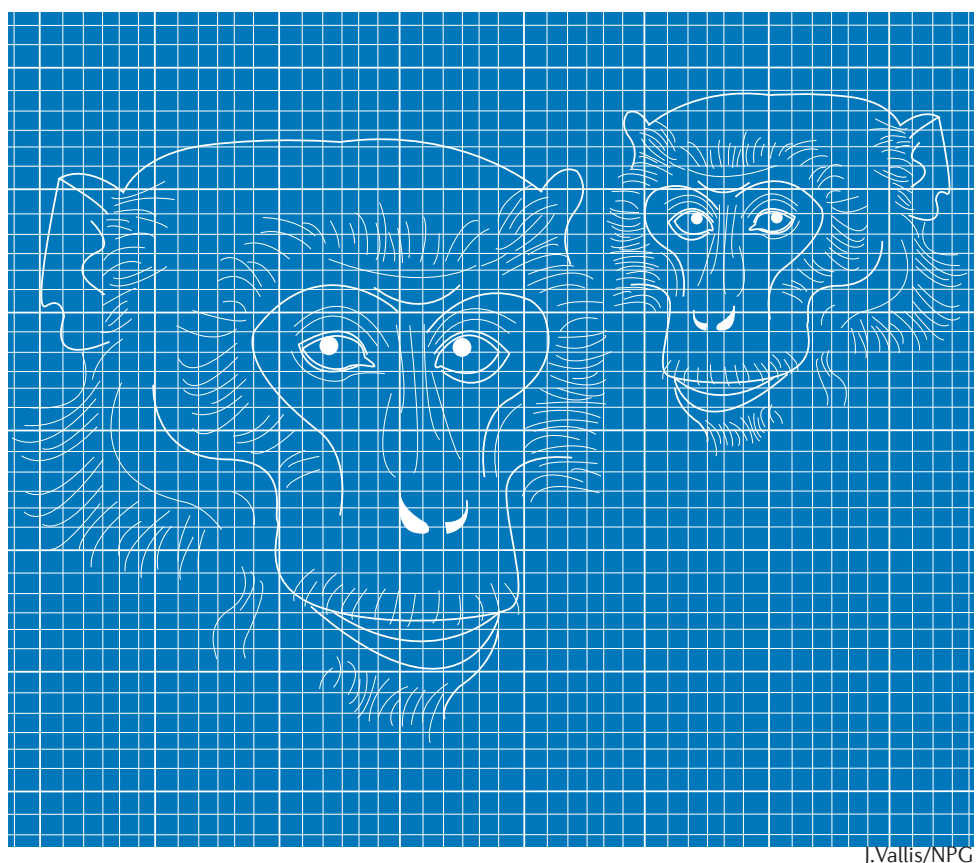

less strongly to repeated presentation of a stimulus - occurred in a larger proportion of anterior than posterior ERC neurons. This points to a possible functional division between these two areas, perhaps similar to the functional distinction between the medial and lateral ERC that has been found in rats. Furthermore, the density of the firing fields decreased as grid cells were located further away from the rhinal sulcus, possibly resembling the dorsal-ventral gradient in firing field spacing in rats.

A recent study showed that in rats, theta oscillations are required to maintain the firing patterns of grid cells. The grid cells in monkeys also showed theta-modulation. Specifically, the cells were most likely to spike during the trough of the theta oscillation. Notably, theta oscillations in the ERC occurred in irregular bouts, and grid cell firing also occurred when no theta rhythm was present. This suggests that, at least in the monkey ERC, an ongoing theta rhythm is not required for grid cell functioning.

This study provides the first direct evidence for the existence of grid cells in the primate ERC. Importantly, the authors showed that these grid cells were active when a monkey was only visually exploring an image, indicating that grid cells may encode spatial locations even in a non-moving animal.

Leonie Welberg

ORIGINAL RESEARCH PAPER Killian, N. J.,

Jutras, M. J. \& Buffalo, E. A. A map of visual space in the primate entorhinal cortex. Nature 28 Oct 2012 (doi:10.1038/nature11587) 